\title{
Veno-arterial extracorporeal membrane oxygenation: Special reference for use in 'post-cardiotomy cardiogenic shock' - A review with an Indian perspective
}

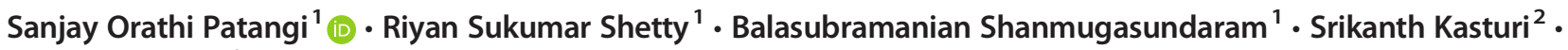 \\ Shivangi Raheja ${ }^{2}$
}

Received: 23 June 2020 / Revised: 14 August 2020 / Accepted: 7 September 2020 / Published online: 7 November 2020

(C) Indian Association of Cardiovascular-Thoracic Surgeons 2020

\begin{abstract}
The ultimate goals of cardiovascular physiology are to ensure adequate end-organ perfusion to satisfy the local metabolic demand, to maintain homeostasis and achieve 'milieu intérieur'. Cardiogenic shock is a state of pump failure which results in tissue hypoperfusion and its associated complications. There are a wide variety of causes which lead to this deranged physiology, and one such important and common scenario is the post-cardiotomy state which is encountered in cardiac surgical units. Venoarterial extracorporeal membrane oxygenation (VA-ECMO) is an important modality of managing post-cardiotomy cardiogenic shock with variable outcomes which would otherwise be universally fatal. VA-ECMO is considered as a double-edged sword with the advantages of luxurious perfusion while providing an avenue for the failing heart to recover, but with the problems of anticoagulation, inflammatory and adverse systemic effects. Optimal outcomes after VA-ECMO are heavily reliant on a multitude of factors and require a multi-disciplinary team to handle them. This article aims to provide an insight into the pathophysiology of VA-ECMO, cannulation techniques, commonly encountered problems, monitoring, weaning strategies and ethical considerations along with a literature review of current evidence-based practices.
\end{abstract}

Keywords Post-cardiotomy cardiogenic shock $\cdot$ Extracorporeal membrane oxygenation

\section{Introduction}

Inability to wean off cardio-pulmonary bypass (CPB) is a morbid condition associated with cardiogenic shock secondary to impaired myocardial contractility. Once the vicious cycle sets in, vital organ perfusion is compromised, culminating in severe metabolic derangement. The incidence of refractory cardiogenic shock post cardiotomy ranges from 0.5 to $6 \%$ $[1,2]$ with a mortality rate as high as $40 \%$ [3]. Veno-arterial extracorporeal membrane oxygenation (VA-ECMO) has gained popularity over the years as a 'bailout' option after

Sanjay Orathi Patangi

sanjaysanjay_op@rediffmail.com

1 Department of Anaesthesia and Intensive Care, Narayana Institute of Cardiac Sciences, 258/A Hosur Road, Bommasandra Industrial Area, Anekal Taluk, Bengaluru, Karnataka 560099, India

2 Department of Cardiothoracic Surgery, Narayana Institute of Cardiac Sciences, Bengaluru, India conventional circulatory support methods have proved refractory in the operating room (OR)/intensive care unit (ICU). VA-ECMO facilitates luxurious end-organ perfusion and adequate gas exchange and supports organ functionality allowing time for recovery/ bridge to decision. However, its usage has not been directly linked with early positive outcomes with few articles reporting increased mortality [4-6]. Post-cardiotomy VA-ECMO is used in both adult and paediatric populations [4].

\section{Background}

The worldwide incidence of instituting post-cardiotomy VAECMO varies between 0.4 and $3.7 \%$ [4]. The Extracorporeal Life Support Organization (ELSO) database states a substantial increase in its use over the last decade. The patient population in which this therapy has been used for dealing with post-cardiotomy cardiogenic shock (PCCS) included those with renal insufficiency, prior myocardial infarction, critical 
left main coronary artery disease, redo-surgery and severe left ventricular (LV) dysfunction [7]. Age is not a contraindication for this therapy. VA-ECMO has been used to tide over patients with PCCS covering the ambit of cardiac surgery and transplantation.

\section{Post-cardiotomy VA-ECMO}

\section{Cannulation}

Cannulation is the first decisive step for a smooth ECMO run that can be either central (atria-aortic) or peripheral (femoral veinfemoral artery/axillary artery) (Fig. 1). A meta-analysis favours the peripheral route due to lesser transfusions, bleeding/ tamponade events and lower mortality [8]. However, these observational studies were based on a small sample size without accounting for confounding factors like the patient pre-initiation condition, effect of LV unloading and the timing of ECMO initiation. Notably, the use of temporary mechanical support following PCCS is associated with higher vascular complications when the duration exceeds 10 days [9] and incidence of amputation even with distal limb perfusion is $3.2 \%$ [10].

\section{Effect of LV venting}

Offloading the LV reduces the myocardial oxygen demand and allows for quicker myocardial recovery with higher survival rates. The decision on cannulation should be expeditious with minimal blood loss ensuring the myocardium is fully rested to hasten recovery and is fundamental in ensuring survival following PCCS. Techniques of venting include insertion of an intra-aortic balloon pump (IABP), atrial septostomy, percutaneous ventricular assist device (VAD) or direct cannulation of the LV apex [11].

\section{Monitoring and maintenance on VA-ECMO}

Standard monitoring includes mean arterial pressure (MAP), central filling pressures, temperature, pulse oximetry and urine

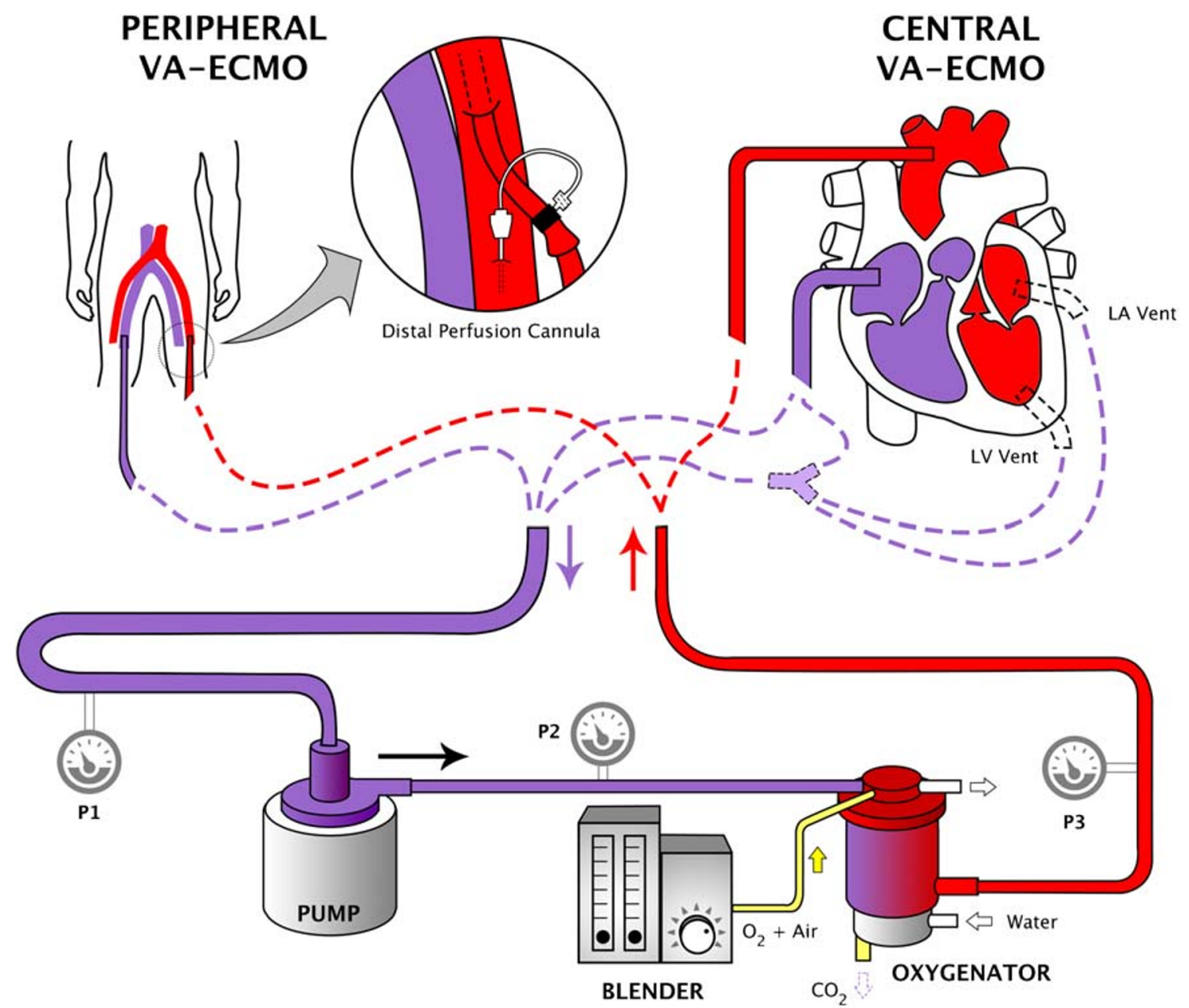

Fig. 1 Cannulation routes and ECMO circuitry. Figure depicting the circuit components of V-A ECMO when used centrally or peripherally with pressure monitors at the pre-pump level (P1), pre-oxygenator level (P2) and post-oxygenator level (P3) 
output. Cardiac output (CO) measurement by thermodilution technique is overestimated whereas pulse contour analysis is unreliable due to non-pulsatility [12]. Abnormal rhythm leads to ineffective LV ejections resulting in LV distension and myocardial injury. Presence of an LV vent prevents distension while reversal to sinus rhythm can be achieved by cardioversion, antiarrhythmics, pacing or ablation [13]. LV pulsatility is assessed by arterial pressure waveform. MAP is maintained in a range of $65-90 \mathrm{mmHg}$ to ensure vital organ perfusion [14].

Temperature monitoring is crucial as ECMO has cooling effects and temperature elevations beyond the 'defined temperature regulated range' signal infection. Vascular access, urinary catheter, pneumonia and surgical wound sites are potential sources of infection [15]. Hypothermia worsens coagulopathy and platelet dysfunction [16]. Transcranial Doppler, near-infrared spectroscopy (NIRS) and cerebral oximetry monitoring can detect cerebral hypoperfusion and upper body hypoxaemia. NIRS helps to identify lower-extremity ischaemia in peripheral arterial cannulation [17]. In VA-ECMO, total systemic flow equals the sum of pump flow plus native $\mathrm{CO}$. Cannulae selected should provide 50 $75 \mathrm{~mL} / \mathrm{kg} / \mathrm{min}$ of flow, and when flows are maintained at $80 \%$, it avoids stasis in the pulmonary vasculature [15]. Sweep gas flow titration regulates carbon dioxide $\left(\mathrm{CO}_{2}\right)$ levels while oxygenation depends on the fraction of delivered oxygen $\left(\mathrm{O}_{2}\right)$, oxygenator blood flow and exposed surface area [13].

VA-ECMO is not an indication for mechanical ventilation, and patients may be extubated on VA-ECMO support [18]. For patients who require mechanical ventilation, there is limited data on optimal ventilation strategies. Extrapolating veno-venous ECMO (VV-ECMO) literature, lung protective ventilation is commonly recommended, with 4-6 $\mathrm{mL} / \mathrm{kg}$ tidal volume, $10 \mathrm{~cm} \mathrm{H}_{2} \mathrm{O}$ positive end expiratory pressure (PEEP) and plateau pressure $<25 \mathrm{cmH}_{2} \mathrm{O}$ to maintain $<15 \mathrm{cmH}_{2} \mathrm{O}$ driving pressure (inspiratory pleural pressure-PEEP) [14]. Unfractionated heparin (UFH) remains the mainstay of anticoagulation due to quick onset of action and rapid reversibility. Heparin monitoring relies on activated partial thromboplastin time (aPTT) and anti-Xa activity while activated clotting time (ACT) is unreliable in low to moderate doses of heparin $(7.5$ to $50 \mathrm{IU} / \mathrm{kg} / \mathrm{h}$ ) [19]. Echocardiography enables assessment of biventricular function, aortic valve opening, cannula position, pericardial and pleural effusions and ventricular thrombus formation [12]. Mixed venous saturations $\left(\mathrm{ScvO}_{2}\right)$ and lactate levels are monitored for adequacy of tissue perfusion and $\mathrm{O}_{2}$ delivery to end organs. Elevated lactate levels reflect tissue hypoxia and are associated with mortality [20]. Plasma free haemoglobin (PFHb) monitoring indicates haemolysis occurring in the ECMO circuit. PFHb levels $>50 \mathrm{mg} / \mathrm{dL}$ increase risk of thrombosis due to affinity of the von Willebrand factor to platelet glycoprotein GPIb [21].

\section{Weaning from ECMO}

No guidelines exist to decide the optimal time of weaning. Recovery of adequate cardiac and respiratory function is a pre-requisite. Some authors suggest weaning as early as 48 $72 \mathrm{~h} \mathrm{[22]} \mathrm{but} \mathrm{rarely} \mathrm{beyond} 15$ days, except in cases of postheart transplantation for resolution of pulmonary hypertension [4]. Recovery of cardiac function in the post-cardiotomy setting is limited and rare beyond 7 days [15, 23]. A longer duration of support is associated with increased complications and mortality [23]. Weaning is by a multidisciplinary consensus. Cardiac index, pulmonary capillary wedge pressure, central venous pressure, pulse pressure $>10 \mathrm{mmHg}$ and MAP $>$ $60 \mathrm{mmHg}$ with minimal haemodynamic support are indicators of cardiac function recovery [24]. Resolution of pulmonary oedema, lung recruitment manoeuvres and clearing of airway secretions by bronchoscopy to ensure partial pressure of $\mathrm{O}_{2}$ $\left(\mathrm{PaO}_{2}\right)$ /fraction of inspired $\mathrm{O}_{2}\left(\mathrm{FiO}_{2}\right)$ ratio $>200, \mathrm{FiO}_{2}<60 \%$ on the ventilator and $\mathrm{FiO}_{2}<50 \%$ on ECMO circuit are desirable $[24,25]$. Although end-organ recovery to pre-ECMO levels is important [15], complete recovery from acute tubular necrosis can take weeks; hence, complete resolution of renal function is not mandatory before weaning. The patient can be supported with haemodiafiltration during this period [26].

\section{Weaning trial}

A weaning trial assesses suitability to separate from the ECMO machine. Assessment of right ventricular (RV) function is crucial [26]. ECMO flows are reduced gradually with inotropic and respiratory support whilst being monitored by clinical and echocardiographic variables. Cavarocchi et al. [27] described a 4-stage weaning trial using a miniaturized transoesophageal echocardiogram (TEE) with $100 \%$ positive predictive value. LV ejection fraction (LVEF) $>20-25 \%$, aortic velocity time integral (VTI) $>10 \mathrm{~cm}$, mitral lateral annulus systolic velocity $>6 \mathrm{~cm} / \mathrm{s}$, RV ejection fraction $>24.6 \%$, no LV or RV distension and ability to maintain MAP with minimal inotropic supports indicate the possibility of a successful wean. Assessments of tricuspid regurgitation, tricuspid annular plane systolic excursion or LV filling parameters are not reliable [24]. Among clinical variables analysed, pulsatility and lactate clearance predicted a successful wean. However, no threshold was identified $[20,26]$. Biomarkers such as Nterminal fragment of the B-type natriuretic peptide (BNP), troponin I, the mid regional fragment of the proatrial natriuretic peptide, proadrenomedullin and copeptin have poor predictive ability in VA-ECMO weaning [28].

\section{Weaning approach}

Fast and slow weaning strategies have been proposed [26, 29]. Westrope et al. described a unique technique of pump- 
controlled retrograde trial off. Here, the pump speed is gradually decreased to encourage reversal of flow into the ECMO circuit which creates a controlled arterio-venous $(\mathrm{A}-\mathrm{V})$ shunt without a steep drop in systemic vascular resistance [30]. The pulmonary artery catheter is a useful monitoring tool in the weaning/post-weaning phase [27]. Levosimendan improves weaning success. It works via non-adrenergic pathways with active metabolites producing effects that last 7-9 days [31]. Inhaled nitric oxide improves RV function by reducing pulmonary vascular resistance [32]. Total vessel density and perfused vessel density monitoring of sublingual microcirculation have been found to have a good association with aortic VTI, LVEF parameters during ECMO wean [33].

\section{Discussion}

PCCS is an uncommon occurrence with high morbidity and mortality in cardiac surgery. This low CO state is refractory to high inotropic and IABP use. In this scenario, VA-ECMO is a bridge to decision/recovery.

\section{Pathophysiology of ECMO}

\section{Cardiovascular system}

VA-ECMO drains blood from the venous system and inputs into the arterial system causing a reduction in RV and LV preload and improving endocardial blood flow by decreasing $\mathrm{LV}$ end diastolic pressure. LV afterload is increased in higher MAP states which distends the LV and leads to pulmonary congestion [34]. The higher flows via ECMO improve macroand micro-circulation [35]. Non-pulsatile flow produces atrophic changes in the medial layer of the aorta and reduces vascular contractility [36].

\section{Respiratory system}

The ECMO oxygenator is made of microporous polypropylene membrane containing hollow fibre bundles. The input gas flows within these bundles while blood passes over it. Gas exchange is by diffusion. The $\mathrm{O}_{2}$ uptake is determined by $\mathrm{FiO}_{2}$, gradient across membrane fibres, surface area of oxygenator and ECMO flow while sweep gas controls $\mathrm{CO}_{2}$ elimination. As $\mathrm{CO}_{2}$ transfer is six times faster compared to $\mathrm{O}_{2}$, failure to clear $\mathrm{CO}_{2}$ indicates oxygenator failure [34]. Ischaemic damage due to shunting of pulmonary blood flow, inflammatory activation, collapsed lungs, ischaemiareperfusion damage and passive congestion from LV distension contribute to ECMO-induced lung damage [37]. Extubation is recommended to reduce lung damage. However, patients have reduced alveolar ventilation secondary to $\mathrm{CO}_{2}$ clearance by ECMO. To prevent post-extubation atelectasis, it is important to maintain $\mathrm{CO}_{2}$ and $\mathrm{pH}$ levels by non-invasive ventilation along with sweep gas flow titration [38]. Although VA-ECMO provides gas exchange in addition to circulatory support, native lung function is important in peripheral ECMO as myocardial and cerebral oxygen delivery is determined by $\mathrm{O}_{2}$ content of blood exiting the LV [39].

\section{Neurological system}

Studies comparing pulsatile with non-pulsatile flows have found maintained cerebral metabolism and autoregulation with both patterns provided MAP is $>50 \mathrm{mmHg}$ [40].

\section{Abdomen}

Varying levels of organ damage prior to ECMO improve after establishing adequate flows. Effective venous drainage relieves congestion, further improving organ circulation. Pulsatile flow enhances end organ recovery and splanchnic circulation compared to non-pulsatile flows although microcirculation is maintained in both patterns [41]. Rate of renal recovery is comparable in either flow patterns [40]. There is no difference in clinical outcomes in either pattern provided adequate flows are maintained [41].

\section{Lymphatic system}

Lymph flow is dependent on muscle activity and pulsatility. Arterial pulsation is a primary determinant of lymphatic drainage in supine patients. Non-pulsatile flows lead to peripheral oedema and intestinal congestion [40].

\section{Inflammatory response}

The blood-material interface activates coagulation, fibrinolytic and inflammatory systems releasing proinflammatory mediators that lead to endothelial injury and neutrophil activation affecting other organ systems. Mast cell degranulation produces vasoplegia requiring vasoconstrictors to maintain MAP [15].

Table 1 Major complications of extracorporeal life support (ECLS)

\begin{tabular}{ll}
$\begin{array}{l}\text { Mechanical (ECLS circuit related) } \\
\text { complications }\end{array}$ & Patient-related complications \\
\hline Clots in the circuit & Surgical site bleeding \\
Air in the circuit & Access site bleeding \\
Oxygenator failure & GI haemorrhage \\
Pump failure & Intracranial haemorrhage \\
Tubing rupture & Cardiac tamponade \\
& Haemolysis \\
\hline
\end{tabular}




\section{Complications of ECMO support}

Major complications can be broadly divided into circuitrelated and patient-related factors (Table 1). The commonest mechanical complication is thrombosis within the circuit [42]. Thrombosis starts in areas of low flow and increases turbulence. Clots on the arterial side of the circuit have a risk of embolization into systemic circulation and should be immediately addressed. Clots on the venous side can cause coating of oxygenator and its failure [43]. Even when visible clots are absent, microthrombi and fibrin deposits on the oxygenator reduce its efficiency over time due to suboptimal gas exchange. This is more common, but not limited to long ECMO runs. Air can enter into the circuit via loosely attached connectors, inadvertently open access ports or tube defects. This can be catastrophic by bringing the pump to a halt. Air on the arterial side can embolize as well. In view of the critical nature of the patient subset undergoing ECMO therapy, complications can have a significant impact on outcomes [44].

\section{Bleeding}

Disseminated intravascular coagulation and acquired von Willebrand disease are seen in patients on ECMO due to activation of the coagulation cascade with resultant consumption coagulopathy with a 10-30\% incidence of bleeding [45]. The commonest sources of bleed are surgical and cannulation sites. Intra-thoracic, intra-abdominal and intra-cranial bleeds can also occur [44]. Incidence of reopening for tamponade or haemorrhagic complications can be as high as $40 \%$, paving the way for low-dose heparin protocols during the maintenance phase of ECMO [46]. Lower antithrombin III levels are associated with higher transfusion requirements and mortality rates [47]. Replacement of blood components is based on haematocrit (hct), ACT, prothrombin time (PT) and aPTT ratios. Point-of-care testing like thromboelastography (TEG) and rotational thromboelastometry allows for quick intervention and specific corrections thereby reducing risk of volume overload and inflammatory/immunological activation. The recommended target ACT is $200-220 \mathrm{~s}$ which is reduced to $170-190 \mathrm{~s}$ in the event of bleeding. An international normalized ratio (INR) of $>1.5$ warrants correction with fresh frozen plasma, and maintaining platelet count of $>50,000 / \mathrm{mm}^{3}$ is recommended. Activated factor VII (VIIa) is used as a last measure when other modalities have failed. Extreme care is exercised when VIIa is used and a lower dose of $25-50 \mu \mathrm{g} / \mathrm{kg}$ is recommended as opposed to a conventional dose of 50$90 \mu \mathrm{g} / \mathrm{kg}$ to avoid an inadvertent pro-thrombotic state [44].

\section{Problems associated with anticoagulation}

UFH is the anticoagulant recommended for initiating and maintaining ECMO support as per 2014 ELSO guidelines
[48]. Heparin-induced thrombocytopenia (HIT) is associated with usage of UFH. HIT is an immune-mediated pro-thrombotic condition characterized by antibodies to the heparin-PF4 complex on platelet surfaces which induces thrombosis. It has an incidence of $0.5-5 \%$ and mortality rate of $42 \%$ [49]. Bivalarudin, a direct thrombin inhibitor, is an alternative anticoagulant for HIT-positive patients. TEG and aPTT ratio are used to monitor anticoagulation [50].

\section{Gastrointestinal complications}

The incidence of gastrointestinal (GI) bleeding is 6-13.5\% secondary to reduced gut perfusion, decreased gastric $\mathrm{pH}$ leading to stress ulcers and $\mathrm{A}-\mathrm{V}$ malformations in the small bowel as a result of non-pulsatile flows [51]. Other contributing factors for GI bleeding include anticoagulation, coagulopathy, thrombocytopenia, platelet dysfunction, acquired von Willebrand syndrome and hyperfibrinolysis. GI bleed commonly occurs around the 11th day of ECMO [52]. Elderly patients and need of high-volume red blood cell transfusion were associated with higher mortality [53]. ELSO registry data showed high mortality from GI bleed in contrast to recent studies [51, 52]. No specific guidelines for prevention of GI bleeding are available. A meta-analysis in critically ill patients showed that proton pump inhibitor prophylaxis reduces incidence of GI bleed, albeit with higher risk of ventilatorassociated pneumonia (VAP) [54]. ELSO guidelines recommend correction of coagulation followed by endoscopy and endotherapy. Endotherapy using haemospray, fibrin glue, cyanoacrylate, cautery and clips has been used to control GI bleeding successfully [51].

\section{Altered liver functions on ECMO}

Hyperbilirubinaemia and elevated liver enzymes are commonly seen in patients on ECMO and are challenging to manage due to limited therapeutic options. Pre-existing liver disease can manifest as acute liver dysfunction on ECMO. When associated with cardiorespiratory problems, passive hepatic congestion can lead to chronic changes and fibrosis [55]. The current concept of 'two hit' ischaemic liver injury happens when a liver primed by such chronic congestion experiences acute hypoperfusion causing a rapid spike in aspartate transaminase (AST) and alanine transaminase (ALT) levels followed by hyperbilirubinaemia [56]. Hyperbilirubinaemia, an independent predictor of poor outcomes, occurs due to a combination of extracorporeal haemolysis and liver dysfunction [57]. Elevated alkaline phosphatase, lactate and BNP are associated with poor outcomes [58], while elevated AST and ALT levels do not seem to be predictive. In patients on ECMO, studies have shown that baseline Model for End stage Liver Disease, United Network for Organ Sharing modification (MELD UNOS) [59] and Model for End Stage Liver 
Disease score excluding INR (MELD XI) [60] scores can predict outcomes. Patients on ECMO can develop acute liver failure (ALF) associated with high mortality. A study has reported that $8 \%$ of extracorporeal life support (ECLS) patients without pre-existing liver disease developed an ALFlike picture [61]. In cases where VA-ECMO was used as a bridge to heart transplant, pre-existing liver dysfunction with total bilirubin $\geq 120 \mu \mathrm{mol} / \mathrm{L}$ and INR $\geq 3.0$ was a predictor of mortality [62]. Similarly, a MELD UNOS score $>24$ has also been noted to be associated with high mortality [59]. Ischaemic hepatitis and liver congestion are self-limiting, and measures which improve cardiac function can restore liver perfusion and aid recovery [55]. Molecular adsorbent recirculation system therapy may be considered in the setting of ALF as it has been noted to accelerate recovery of liver function and improve survival [63].

\section{Renal complications}

Acute kidney injury (AKI) is seen in $85 \%$ of patients undergoing ECMO with complex multifactorial aetiology and pathophysiology with majority of them requiring renal replacement therapy (RRT) [64]. The most common indication for initiating RRT is to achieve fluid balance in patients unresponsive to diuretic therapy. Intermittent therapies of RRT are effective in haemodynamically stable patients; however, the most common modality used is continuous RRT (CRRT). A large meta-analysis has shown higher mortality when RRT was used sparingly in patients on ECMO [65]. RRT can be provided using either an 'integrated system' or a 'parallel system'. The integrated system could be an 'in-line' haemofilter or an RRT circuit incorporated into the ECMO circuit. However, when an integrated RRT circuit is used, there is a risk of micro-clot formation within the circuit clogging the oxygenator. Hence, knowledge of intra-circuit pressures, appropriate modifications in connections of the RRT circuit and additional regional anticoagulation would be necessary. On the other hand, a 'parallel system' involves a separate indwelling vascular catheter to provide RRT, obliviating the need for additional anticoagulation as patients are already anticoagulated for ECMO. Close review and readjustment of prescription is crucial as per changing needs of the patient. Most studies have shown that use of CRRT with ECMO is not associated with increased mortality, and in fact, when used, these subset of patients needed shorter duration of ECMO. Data from large ECMO centres have shown that ECMO survivors who have received RRT have similar renal outcomes with no increase in incidence of endstage renal disease in comparison to patients who did not receive RRT [66].

\section{Neurological complications}

Neurological complications in VA-ECMO are associated with high mortality and morbidity, with high incidence (7.4-15\%) across all age groups [67]. This has been attributed to nonpulsatile flow, low arterial $\mathrm{O}_{2}$ saturation in the upper half of the body and entrainment of unfiltered thrombi into the systemic circulation [68]. Risk factors associated with neurological injury in neonates are low birth weight $<3 \mathrm{~kg}$, gestational age $<34$ weeks, pre-ECMO cardio-pulmonary resuscitation (CPR), metabolic acidosis, bicarbonate use and prior ECMO exposure [69]. Neonates are more susceptible to intracranial haemorrhage $(\mathrm{ICH})(1.4 \%)$ as opposed to the paediatric group $(0.9 \%)$ and adults $(0.4 \%)$ [70]. Risk factors in adults include female gender, central cannulation during cardiac surgery, thrombocytopenia, serum creatinine $>2.6 \mathrm{mg} / \mathrm{dL}$, hypercapnia while initiating ECMO, duration of ECMO and use of anticoagulants. Incidence of acute ischaemic stroke is 3.6$6 \%$ across all age groups which is multifactorial in origin [68]. Thrombocytosis at ECMO initiation is a potentially modifiable factor [67]. Brain computed tomography scan (Fig. 2) is the recommended imaging modality when neurological deficits are identified since magnetic resonance imaging, despite being more sensitive, is contraindicated during ECMO [68]. Use of VA-ECMO is associated with a higher incidence of electrographic seizures (Fig. 3) in neonates $(4.9 \%)$ and children $(3.3 \%)$ as compared to adults $(0.5 \%)$ [70].

\section{Infections}

Nosocomial infection is a major cause of morbidity and mortality on ECMO, with an incidence of 11-64\% [71-73]. The

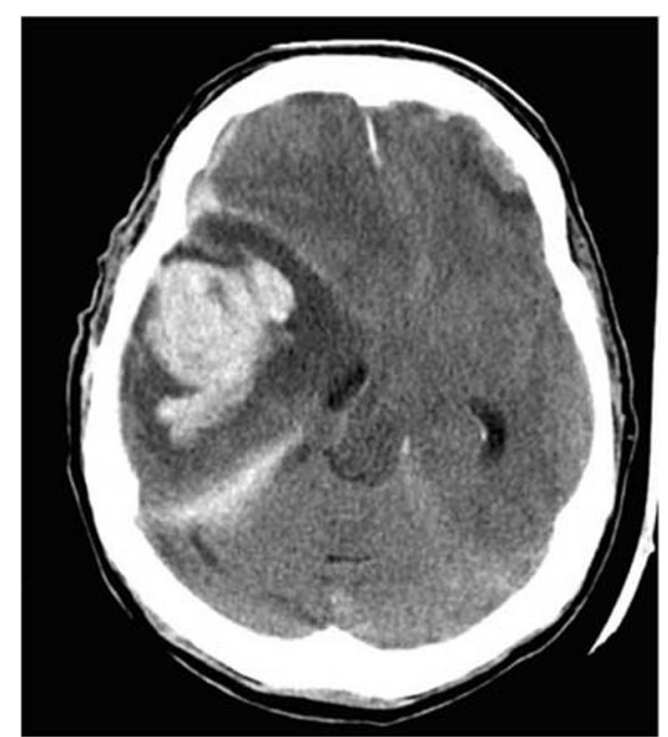

Fig. 2 Non-contrast computed tomography of the brain showing rightsided intra-parenchymal haemorrhage with peri-haemorrhagic cerebral oedema and midline shift on day 2 of starting VA ECMO 
rate of infection was highest in adults, followed by paediatric and neonatal age groups $[71,72]$. It is crucial to practice meticulous infection prevention measures. Longer duration of ECMO support is an independent risk factor for infection [73]. Bizzarro et al. reported a prevalence of $30.3 \%$ in patients on ECMO > 14 days compared to $6.1 \%$ in patients with $<$ 7 days support [71]. Bloodstream infections and VAP were commonly encountered [74]. The median time interval between initiation of ECMO and occurence of a bloodstream infection was 5-8 days [72]. These infections were predominantly caused by gram-positive organisms (coagulase-negative Stapylococcus, Enterococcus and Staphylococcus aureus), Candida and Pseudomonas species [71, 73].

\section{Vascular complications}

Vascular complications stem from difficult cannulation, low flow states and high use of vasoconstrictors. Percutaneous cannulation techniques in peripheral VA-ECMO can be associated with posterior vessel wall perforation resulting in inadequate perfusion and subsequent development of a compartment syndrome/retro-peritoneal haematoma [75]. Larger-size cannulae (>20F) usage, female gender and associated peripheral vascular disease are proven risk factors [44]. Insertion of a distal perfusion cannula should be considered to augment perfusion [76].

\section{Other complications}

Differential hypoxia, North-South/Harlequin syndrome, occurs in peripheral VA-ECMO when the heart has recovered on the backdrop of a lung still lagging behind. The peripheral
ECMO cannot compete with native $\mathrm{CO}$, which causes poorly oxygenated blood supply to the upper half of the body while the lower half of the body receives well-oxygenated blood from the circuit [44]. Monitoring the patient's arterial saturation in the right upper limb helps in diagnosis. Remedial measures include advancement of inferior vena caval cannula and delivery of oxygenated blood into the right atrium by venoarterial-venous ECMO/ hybrid circuit [77].

\section{ECMO in the paediatric age group}

The incidence of PCCS VA-ECMO is $2.2 \%$ in paediatric cardiac surgery [78]. Operative stress and residual lesions added on to a physiologically compromised heart in congenital heart disease can predispose to poor cardiac function post-operatively. There is no consensus on the timing to initiate ECMO in the paediatric population. The indications and contraindications of post-cardiotomy ECMO are listed in Table 2.

\section{Pathophysiology of paediatric ECMO}

Oxygen extraction ratio $\left(\mathrm{O}_{2} \mathrm{ER}\right)$ is the ratio between oxygen consumption $\left(\mathrm{VO}_{2}\right)$ and $\mathrm{O}_{2}$ delivery $\left(\mathrm{DO}_{2}\right)$. Normal $\mathrm{O}_{2} \mathrm{ER}$ is 1:5 or $20 \%$, derived from $\mathrm{ScvO}_{2} . \mathrm{DO}_{2}$ reduces in low perfusion states. Up to a certain point, tissues maintain aerobic metabolism by extracting $\mathrm{O}_{2}$ from blood, increasing the central venous extraction and $\mathrm{O}_{2}$ ER. At an $\mathrm{O}_{2}$ ER of $1: 2$, there is an imbalance between metabolic demand and aerobic metabolism thereby initiating anaerobic metabolism, producing lactate and metabolic acidosis. $\mathrm{O}_{2}$ consumption in infants and children $(5-7 \mathrm{~mL} / \mathrm{kg} / \mathrm{min})$ is more in comparison to that in
Fig. 3 An excerpt of continuous EEG monitoring of the patient depicted in Fig. 2 showing right hemispheric epileptic activity

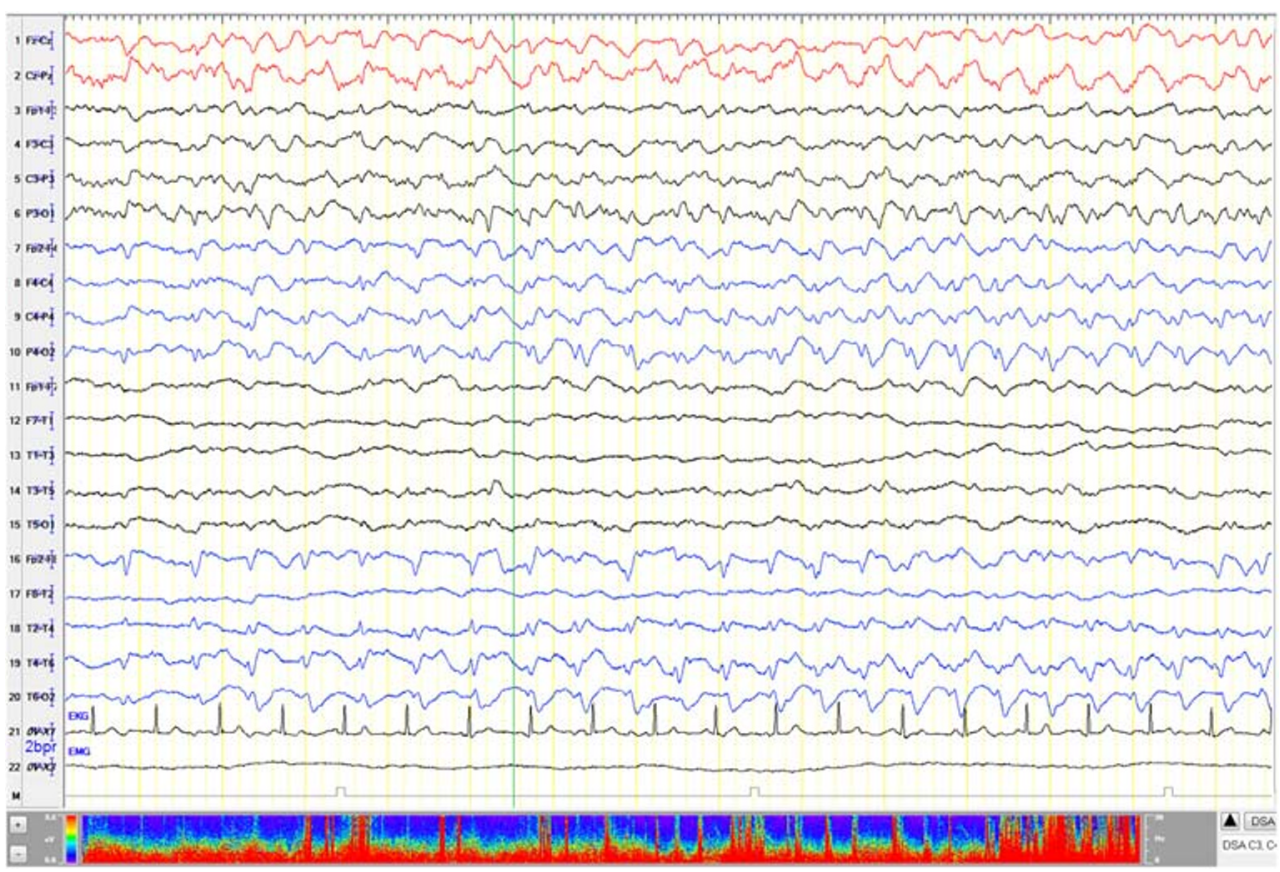


Table 2 Indications and contra-indications of post-cardiotomy ECMO

\section{Indications \\ Failure to wean from $\mathrm{CPB}$ \\ Refractory low CO \\ Uncontrolled pulmonary hypertension \\ Postoperative intractable arrhythmias \\ Cardiac arrest not responsive to conventional CPR (extracorporeal cardiopulmonary resuscitation or ECPR) \\ Contraindications \\ Lethal chromosomal abnormalities \\ Severe irreversible brain injury \\ Extremely low gestational and weight $(<32$ weeks gestation or $<1.5 \mathrm{~kg}$ ) \\ Uncontrollable haemorrhage}

adults (3-4 $\mathrm{mL} / \mathrm{kg} / \mathrm{min})$; hence, anaerobic metabolism and organ damage occur earlier [79].

\section{Anticoagulation in paediatric ECMO}

UFH is the mainstay for anticoagulation in children on ECMO. As HIT is rare in children [80], the need for alternative anticoagulants seldom arises. ELSO guidelines [81] suggest a bolus dose of 50-100 units/kg of UFH before cannulation and an infusion of $10-40 \mathrm{units} / \mathrm{kg} / \mathrm{h}$ for maintenance. Besides ACT, aPTT ratio, TEG, antithrombin and activated factor Xa levels are used for titration of anticoagulant.

\section{Management on ECMO}

Transthoracic echocardiography (TTE), and catheterbased diagnostic studies aid in detection of postoperative residual lesions which, when addressed, help in weaning from ECMO [82]. The decision to vent the heart is based on TTE findings. TTE assists in documenting serial ventricular function improvement, identifying pericardial/ pleural effusions, assessing pulmonary hypertension and shunting at atrial or ventricular levels [83]. All inotropes are stopped when complete myocardial rest is indicated. MAP targets are achieved using vasoconstrictors and vasodilators. Although there is limited experience, levosimendan $(0.1-0.2 \mathrm{mcg} / \mathrm{kg} / \mathrm{min}$ for $24 \mathrm{~h})$ can be used during ECLS to aid weaning [84]. MAP, capillary refill time, urine output, lactate levels and $\mathrm{ScvO}_{2}$ trends are reliable monitoring tools. A hct of $35 \%$ is targeted if $\mathrm{ScvO}_{2}>75 \%$. In situations of $\mathrm{DO}_{2} / \mathrm{VO}_{2}$ mismatch/ palliated single-ventricle patients, a higher hct $(>40 \%)$ is targetted. Gentle ventilation to achieve $5-6 \mathrm{~mL} / \mathrm{kg}$ tidal volume and a PEEP of up to $10 \mathrm{mmHg}$ is preferred to avoid barotrauma [81]. ECMO circuitry triggers an inflammatory response and capillary leak, causing fluid to

shift out of the intravascular compartment. In the backdrop of intravascular volume depletion secondary to bleeding, patients are predisposed to prerenal AKI aggravating a pre-existing hypoxic kidney injury. Once volume status is normalized within $48-72 \mathrm{~h}$, the capillary leak subsides and diuresis improves [85]. Treatment options for inadequate diuresis are diuretics, peritoneal dialysis (PD) and modified ultrafiltration on the circuit. RRT has not shown to improve mortality in children on ECMO [86].

Bowel hypoperfusion secondary to low $\mathrm{CO}$, sedative and paralytic agent usage, vasoconstrictive drugs and gut inflammation can predispose to gastric dysmotility and feed intolerance in a child on ECMO [87]. Regional GI ischaemia causes hyperlactaemia. Withholding enteral feeds translocates gut bacteria thereby increasing the risk of sepsis. Starting of trophic feeds once lactate has normalized is a recommended strategy [88]. Parenteral nutrition is considered when there is persistent hyperlactataemia or feed intolerance taking on board the risk of infection [87].
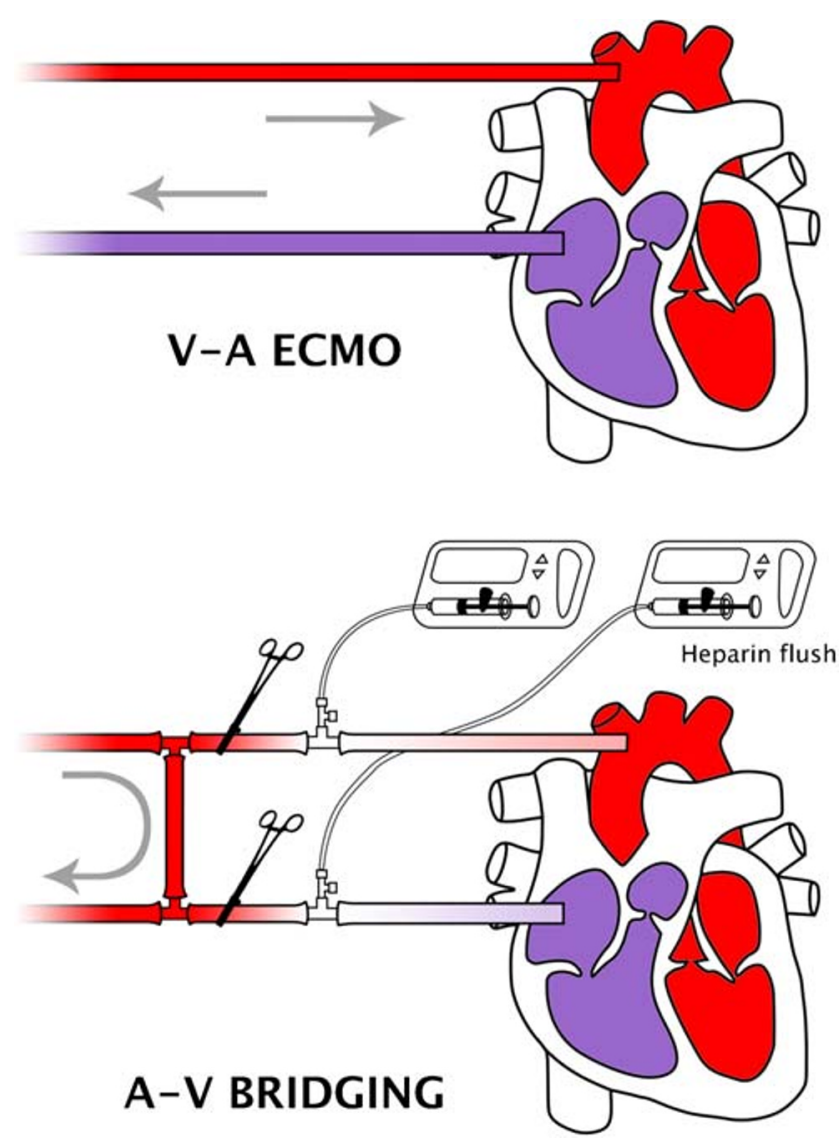

Fig. 4 A-V bridging on ECMO consists of a circuit that runs parallel to the patient with the cannulae in situ but clamped. Heparin flush is constantly circulated through the cannulae during the clamped interval to prevent clotting. If there is haemodynamic or respiratory instability, VA-ECMO is recommenced by just removing the clamps on the venous and arterial cannulae 


\section{Weaning paediatric hearts from ECMO}

The timing and sequence of weaning off ECMO is not standardized. Once the myocardium shows signs of recovery, organ functions have improved and residual lesions are ruled out or corrected, full ventilatory support is commenced, inotropes started to augment cardiac contractility and ECMO flows are gradually reduced. The left-sided vent, if present, is removed when sustained ejections are seen. On further weaning to minimal ECMO flows ( $200 \mathrm{~mL} / \mathrm{min})$, TTE is done. Decision is then taken to decannulate the patient. In borderline cases, A-V bridging with a trial period off ECMO $(1-4 \mathrm{~h})$ is an option. This consists of a circuit that runs parallel to the patient with the cannulae in situ but clamped (Fig. 4). During A-V bridging, if there is haemodynamic or respiratory instability, VA-ECMO is recommenced. If the trial period off ECMO is uneventful, the patient is decannulated [89]. TTE plays a pivotal role during various stages of weaning and in the post-wean phase [83].

\section{Special considerations in patients with functionally univentricular hearts}

Palliative surgery for univentricular hearts poses special challenges on ECMO. In infants with single ventricular physiology and systemic to pulmonary shunts, the shunt has to be partially occluded to counter pulmonary steal. The alternative is to target higher flows [90]. The success rate for ECLS, post Glenn and Fontan procedures is lower. The reasons are multifactorial, including complex physiology and altered cardiac anatomy, the presence of atrioventricular valve regurgitation, previous surgeries, the need for multiple drainage cannulae and the inability to achieve full flows on ECMO [91].

\section{Survival and outcomes}

Success after ECMO typically is defined as survival to hospital discharge after a successful wean [26]. For PCCS VA-ECMO, the average survival rate across all age groups is $46.3 \%$ [70]. The indicators of poor outcome have been outlined as advanced age, redo-valve surgery and climbing lactate levels. Pre-operative pulmonary hypertension had no bearing on mortality [92]. The 5 -year survival rate was $56 \%$ for patients discharged from the hospital while $63 \%$ for patients surviving at 30 days [93]. Discharge from hospital is the most important predictor of successful outcome [2]. Lai et al. concluded that a small percentage of patients especially in a high-volume centre would benefit from additional ECMO therapy if their cardiopulmonary function declined. Additionally, a higher incidence of infection and need for RRT was reported in this subset [93]. Literature per se is nebulous about the quality-of-life predictors after ECMO. Studies have shown 15\% mortality within 3 months of hospital discharge and this increases to $24 \%$ over a 3 -year period [94]. On the contrary, studies have depicted $15.7 \%$ survival at 18 months without elaborating on the quality of life [95]. The New York Heart Association (NYHA) class is a good tool for ascertaining functional status in ECMO survivors [96]. Functionality status of survivors have been diverse, ranging from NYHA class I-IV indicating survivors can achieve a reasonable quality of life. Chen et al. have reported a higher readmission rate during the first year of follow-up resulting in higher medical expenditure. Infections and cardiac events were stated as common causes of mortality/morbidity in the follow-up period [97].

\section{Cost implications}

Literature has extensively debated on the cost-effectiveness of ECMO. Several analyses have deemed ECMO to be an expensive therapy amounting to an average of $\$ 92,600$ per case in the USA and have recommended package payments to buoy individual institutes delivering this service [98]. In the UK, mechanical support is funded only when it has been advocated as a bridge to transplantation [99]. There has been no such cost analysis done in the Indian subcontinent. The average cost of initiating ECMO in India is meagre compared to the west. However, additional costing gets added on to patient maintenance in the ICU, which could include RRT, medication, imaging, laboratory and transfusion costs. By advocating a wholesome package module, the implications of cost can be negated with the outcome benefits.

\section{Ethical considerations}

From an ethical viewpoint, three perspectives have to be considered: surgeons, the patient's family and financial implications. A surgeon would look forward to successful outcomes, but, when faced with a situation of inability to wean off CPB, it is normal to consider options for recovery even if chances of successful outcome is low. The patient's family should be briefed about the condition and given an opportunity to partake in the decision-making. Financial implications of ECMO need be explained as healthcare in India is not funded. Though the cost of initiation of ECMO is finite, duration and end-point are difficult to ascertain and costs are impractical to calculate. There are situations where hospitals or other payers like insurance will meet the additional expense, but that is the exception rather than the rule. These nuances should be discussed and documented to enable the family to make an informed decision. Counselling and communication is key to supporting the family through this tumultuous phase.

\section{ECMO in the Indian subcontinent}

VA-ECMO has made inroads into the management of PCCS in India. Bearing its cost implications, it has yet to make a significant impact in the management of these subset of patients. At Narayana Institute of Cardiac Sciences (NICS), Bangalore, 
India, we on an average perform about 110-115 ECMO runs a year, a majority of which are post-cardiotomy ECMO runs.

Considering the factors influencing cannulation, our institutional preference is the central route. If the patient presents with PCCS, our cannulation sites are the right atrial appendage and the ascending aorta close to the sino-tubular junction. We have observed Harlequin syndrome in those patients with high aortic cannulation, and these patients presented with persistent ventricular fibrillation and altered sensorium. This was due to deoxygenated blood from the LV selectively streaming into coronaries and the right innominate artery. This was promptly reversed by changing to a lower cannula position. Cannulae are snugged using rubber spigots, following which they are tunnelled sub-xiphoid and chest closed with sternal wires. Absolute haemostasis is imperative. A common bleeding point is the aortic cannulation site. For this, 2 rows of purse strings are used that encompass the cannula twice causing a cuff of adventitia to evert around the cannula to stop further bleeding.

For LV venting, our strategy is to cannulate the LV apex directly with a separate limb to the inflow circuit (Fig. 1). We keep a dedicated flow sensor on this limb to detect low flow. An obvious disadvantage of this is formation of a LV clot around the cannula inflow. This is prevented by maintaining a higher aPTT ratio and using a larger-sized cannula. The decision to vent the left heart is based upon the disappearance of ejections on the arterial trace, presence of spontaneous echo contrast on TEE, inadequate flows with obvious signs of LV distention and to prevent Harlequin syndrome in peripheral cannulation. We strongly believe in central cannulation for the following reasons:

- Ensure maximal flow using the largest possible cannula.

- Unload the left heart with easy access to venting sites.

- Convert CPB cannulae to ECMO cannulae, tunnel lines and close the chest. The bleeding is less when compared to an open chest as the marrow is approximated.

- With the chest closed, the patient can be extubated and ambulated.

- Easy conversion of ECMO to temporary VAD support.

At our institute, the weaning process starts in the ICU and the final stage happens in the OR under TEE guidance. The patient is primed with levosimendan overnight without a loading dose. The ventilator is adjusted for optimal gas exchange. Haemodynamic variables are monitored using echocardiography and a pulmonary artery catheter. Metabolic stability is monitored by trends in lactate level, base excess in arterial gases and $\mathrm{ScvO}_{2}$ monitoring. Anticoagulation is maintained with aPTT 45-60 s or ACT 200-220 s. Flows are reduced by $0.5 \mathrm{~L} / \mathrm{min}$ every 2 nd hourly till a flow of $30 \%$ is achieved. If a $\mathrm{LV}$ vent is in situ, a gated clamp regulates the LV vent flows. Flows are reduced in aliquots of $200 \mathrm{~mL}$ every second hour till
Table 3 Our experience with ECMO complications

\begin{tabular}{lll}
\hline & Adult & Paediatric \\
\hline Total cardiac surgeries (2018-2019) & 10,715 & 4765 \\
No. of PCCS VA ECMO & $45(0.42 \%)$ & $120(2.52 \%)$ \\
Average mediastinal drainage on ECMO & \\
a. 8 h post-initiation & $1100 \mathrm{~mL}$ & $275 \mathrm{~mL}$ \\
b. Following 16 h & $868 \mathrm{~mL}$ & $215 \mathrm{~mL}$ \\
Acute kidney injury (AKI) & & \\
a. Total no. of cases & $35(77.77 \%)$ & $26(21.66 \%)$ \\
b. AKI on RRT & $24(53.33 \%)$ & $19(15.83 \%)$ \\
Hyperbilirubinaemia & $20(44.44 \%)$ & $31(25.83 \%)$ \\
Limb ischaemia-total no. of cases & $4(8.88 \%)$ & $4(3.33 \%)$ \\
a. Surgical management & $3(6.66 \%)$ & $2(1.66 \%)$ \\
b. Conservative management & $1(2.22 \%)$ & $2(1.66 \%)$ \\
Neurological complications & & \\
a. Patients with ICH & $5(11.11 \%)$ & $3(2.5 \%)$ \\
b. Brain dead & $2(4.44 \%)$ & Nil \\
c. HIE & $3(6.66 \%)$ & $1(0.83 \%)$ \\
d. Convulsions & Nil & $1(0.83 \%)$ \\
GI complications-total no. of cases & $4(8.88 \%)$ & Nil \\
a. Upper GI bleed & $1(2.22 \%)$ & \\
b. Lower GI bleed & $2(4.44 \%)$ & \\
c. upper and lower GI bleed & $1(2.22 \%)$ & \\
\hline
\end{tabular}

$P C C S$ post-cardiotomy cardiogenic shock, VA ECMO veno-arterial extracorporeal membrane oxygenator, $R R T$ renal replacement therapy, $I C H$ intra-cranial haemorrhage, HIE hypoxic ischaemic encephalopathy, GI gastro-intestinal

a flow of $500 \mathrm{~mL}$ is reached. The patient is idled at this flow till transfer to the OR for final wean and decannulation. Weaning is aborted at any stage if there is ventricular distension, increase in inotropic levels to maintain haemodynamics, worsening gas exchange or metabolic parameters.

\section{Our results}

We performed a total of 15,480 cardiac surgeries in 2018 and 2019. PCCS VA-ECMO was instituted in 165 (1.06\%) of these patients. Our results are broadly outlined in Table 3. Bleeding was a significant problem. Major transfusions were needed in the initial 24-h period. The transfusion requirements were monitored with 4th hourly clotting screens and TEG. The incidence of AKI with RRT requirements were comparable across age groups which is similar to published data [65]. Our preference for instituting RRT is using a parallel circuit to prevent possible air embolism. RRT in the paediatric patients was provided by PD. A rising trend of total bilirubin was associated with failure to wean and mortality. Overall mortality of ECMO patients with limb ischaemia was $87.5 \%$. Literature has shown higher incidence of neurological 
Table 4 Timing of institution of ECMO and corresponding outcomes at our centre

\begin{tabular}{llll}
\hline & Number of patients & Weaned off & Survived to discharge \\
\hline Adult ECMO & & & \\
ECMO from OR & 21 & $9(42.85 \%)$ & $2(9.52 \%)$ \\
Semi-elective & 6 & $4(66.66 \%)$ & $3(50 \%)$ \\
Emergency & 18 & $5(27.77 \%)$ & $3(16.66 \%)$ \\
Paediatric ECMO & & & $9(26.47 \%)$ \\
ECMO from OR & 34 & $16(47.05 \%)$ & $30(65.22 \%)$ \\
Semi-elective & 46 & $38(82.61 \%)$ & $13(32.5 \%)$ \\
Emergency & 40 & $22(55 \%)$ & \\
\hline
\end{tabular}

$E C M O$ extracorporeal membrane oxygenator, $O R$ operating room complications in paediatric ECMO [67-69] in contrast to our data. The incidence of GI bleed in our cohort was $2.42 \%$ which compares favourably with published data $[52,54]$.

Better weaning results were observed between 2018 and 2019 (Table 4) in the adult group (32\% and 45\% in 2018 and 2019 respectively) which we attribute to the following factors:

- Early institution of VA-ECMO in the postoperative period

- Apical LV venting

- Early institution of RRT for fluid balance and metabolic derangement

- Use of levosimendan during initiation of wean

A review of the bloodstream infections in patients on PCCS VA-ECMO revealed a predominance in gramnegative bacilli (GNB) (85\%), followed by candida species (10\%) and gram-positive organisms (5\%). Klebsiella species constituted the majority of the GNB bacteraemias. Carbapenem resistance was present in $44 \%$ of the gramnegative organisms isolated (65\% carbapenem resistance in Klebsiella species alone). Most of the blood culture isolates grew within the first $72 \mathrm{~h}$ of incubation. However, $13 \%$ of the organisms grew only after 5 days of incubation, highlighting the importance of incubating the blood culture for a minimum of 7 days and monitoring them for growth.

\section{Conclusion}

PCCS VA-ECMO has achieved a 'niche' in cardiac surgery as a bridge to decision/recovery. Although an expensive and demanding therapy, it is versatile and can save lives if incorporated in a timely fashion. The positive outcomes are driven by the experience of the team handling the case. Over time, PCCS VA-ECMO has achieved a 'standard of care' status. Since resource utilization for this therapy is high, a scoring system is warranted to ascertain which subset of patients would benefit and have a positive outcome.
Acknowledgements We would like to acknowledge the help and insights provided by Dr. Julius Punnen, Dr. Varun Shetty, Dr. Rammohan Sripad Bhat, Dr. Vijay Samuel Richard, Dr. Rangarajan Kasturi, Dr. Radhika Manohar, Dr. Shrinivas Hittalamani and Dr. Anup Daniel Varghese who are specialists in their fields in writing this document. We would also like to mention the assistance provided by Mr. Selvakumar, Senior Perfusionist and the Department of Perfusion Technology, Narayana Institute of Cardiac Sciences, Bengaluru, for help with data collation for this manuscript.

Funding None.

Data availability Not applicable.

\section{Compliance with ethical standards}

Conflict of interest The authors declare that they have no competing interests.

Code availability Not applicable.

\section{References}

1. Fux T, Holm M, Corbascio M, Lund LH, van der Linden J. Venoarterial extracorporeal membrane oxygenation for postcardiotomy shock: Risk factors for mortality. J Thorac Cardiovasc Surg. 2018;156:1894-1902.e3.

2. Meani P, Matteucci M, Jiritano F, et al. Long-term survival and major outcomes in post-cardiotomy extracorporeal membrane oxygenation for adult patients in cardiogenic shock. Ann Cardiothorac Surg. 2019;8:116-22.

3. Bellumkonda L, Gul B, Masri SC. Evolving concepts in diagnosis and management of cardiogenic shock. Am J Cardiol. 2018;122: 1104-10.

4. Lorusso R, Raffa GM, Alenizy K, et al. Structured review of postcardiotomy extracorporeal membrane oxygenation: part 1-adult patients. J Heart Lung Transplant. 2019;38:1125-43.

5. Whitman GJR. Extracorporeal membrane oxygenation for the treatment of postcardiotomy shock. J Thorac Cardiovasc Surg. 2017;153:95-101.

6. Fukuhara S, Takeda K, Garan AR, et al. Contemporary mechanical circulatory support therapy for postcardiotomy shock. Gen Thorac Cardiovasc Surg. 2016;64:183-91. 
7. Smedira NG, Blackstone EH. Postcardiotomy mechanical support: risk factors and outcomes. Ann Thorac Surg. 2001;71:S60-6.

8. Mariscalco G, Salsano A, Fiore A, et al. Peripheral versus central extracorporeal membrane oxygenation for postcardiotomy shock: Multicenter registry, systematic review, and meta-analysis. J Thorac Cardiovasc Surg. 2019;:S0022-5223(19):32376-1. https:// doi.org/10.1016/j.jtcvs.2019.10.078.

9. Ganslmeier P, Philipp A, Rupprecht L, et al. Percutaneous cannulation for extracorporeal life support. Thorac Cardiovasc Surg. 2011;59:103-7.

10. Slottosch I, Liakopoulos O, Kuhn E, et al. Outcomes after peripheral extracorporeal membrane oxygenation therapy for postcardiotomy cardiogenic shock: a single-center experience. J Surg Res. 2013;181:e47-55.

11. Donker DW, Brodie D, Henriques JPS, Broomé M. Left ventricular unloading during veno-arterial ECMO: a review of percutaneous and surgical unloading interventions. Perfusion. 2019;34:98-105.

12. Douflé G, Roscoe A, Billia F, Fan E. Echocardiography for adult patients supported with extracorporeal membrane oxygenation. Crit Care. 2015;19:326.

13. Chung M, Shiloh AL, Carlese A. Monitoring of the adult patient on venoarterial extracorporeal membrane oxygenation. Scientific World J. 2014;2014:393258.

14. Hoyler MM, Flynn B, Iannacone EM, Jones M-M, Ivascu NS. Clinical management of venoarterial extracorporeal membrane oxygenation. J Cardiothorac Vasc Anesth. 2020; S10530770(20)30017-3.

15. Brogan TV. Extracorporeal life support: the ELSO red book. Ann Arbor: Extracorporeal Life Support Organization; 2017.

16. Van Poucke S, Stevens K, Marcus AE, Lancé M. Hypothermia: effects on platelet function and hemostasis. Thromb J. 2014;12:31.

17. Kim HS, Ha SO, Yu K-H, et al. Cerebral oxygenation as a monitoring parameter for mortality during Venoarterial extracorporeal membrane oxygenation. ASAIO J. 2019;65:342-8.

18. Abrams D, Garan AR, Brodie D. Awake and fully mobile patients on cardiac extracorporeal life support. Ann Cardiothorac Surg. 2019;8:44-53.

19. Ranucci M, Baryshnikova E, Cotza M, et al. Coagulation monitoring in postcardiotomy ECMO: conventional tests, point-of-care, or both? Minerva Anestesiol. 2016;82:858-66.

20. Li C-L, Wang H, Jia M, Ma N, Meng X, Hou X-T. The early dynamic behavior of lactate is linked to mortality in postcardiotomy patients with extracorporeal membrane oxygenation support: a retrospective observational study. J Thorac Cardiovasc Surg. 2015;149:1445-50.

21. Valladolid C, Yee A, Cruz MA. von Willebrand factor, free hemoglobin and thrombosis in ECMO. Front Med (Lausanne). 2018;5: 228.

22. Fiser SM, Tribble CG, Kaza AK, et al. When to discontinue extracorporeal membrane oxygenation for postcardiotomy support. Ann Thorac Surg. 2001;71:210-4.

23. Distelmaier K, Wiedemann D, Binder C, et al. Duration of extracorporeal membrane oxygenation support and survival in cardiovascular surgery patients. J Thorac Cardiovasc Surg. 2018;155: 2471-6.

24. Aissaoui N, El-Banayosy A, Combes A. How to wean a patient from veno-arterial extracorporeal membrane oxygenation. Intensive Care Med. 2015;41:902-5.

25. Zwischenberger JB, Pitcher HT. Extracorporeal membrane oxygenation management: techniques to liberate from extracorporeal membrane oxygenation and manage post-intensive care unit issues. Crit Care Clin. 2017;33:843-53

26. Ortuno S, Delmas C, Diehl J-L, et al. Weaning from veno-arterial extra-corporeal membrane oxygenation: which strategy to use? Ann Cardiothorac Surg. 2019;8:E1-8.
27. Cavarocchi NC, Pitcher HT, Yang Q, et al. Weaning of extracorporeal membrane oxygenation using continuous hemodynamic transesophageal echocardiography. J Thorac Cardiovasc Surg. 2013;146:1474-9.

28. Luyt C-E, Landivier A, Leprince P, et al. Usefulness of cardiac biomarkers to predict cardiac recovery in patients on extracorporeal membrane oxygenation support for refractory cardiogenic shock. J Crit Care. 2012;27:524.e7-14.

29. Keebler ME, Haddad EV, Choi CW, et al. Venoarterial extracorporeal membrane oxygenation in cardiogenic shock. JACC Heart Fail. 2018;6:503-16.

30. Westrope C, Harvey C, Robinson S, Speggiorin S, Faulkner G, Peek GJ. Pump controlled retrograde trial off from VA-ECMO. ASAIO J. 2013;59:517-9.

31. Affronti A, di Bella I, Carino D, Ragni T. Levosimendan may improve weaning outcomes in venoarterial ECMO patients. ASAIO J. 2013;59:554-7.

32. Cui WW, Ramsay JG. Pharmacologic approaches to weaning from cardiopulmonary bypass and extracorporeal membrane oxygenation. Best Pract Res Clin Anaesthesiol. 2015;29:257-70.

33. Akin S, Dos Reis MD, Caliskan K, et al. Functional evaluation of sublingual microcirculation indicates successful weaning from VAECMO in cardiogenic shock. Crit Care. 2017;21:265.

34. Choi MS, Sung K, Cho YH. Clinical pearls of venoarterial extracorporeal membrane oxygenation for cardiogenic shock. Korean Circ J. 2019;49:657-77.

35. Chauhan S, Subin S. Extracorporeal membrane oxygenation, an anesthesiologist's perspective: physiology and principles. Part 1. Ann Card Anaesth. 2011;14:218-29.

36. Nishimura T, Tatsumi E, Nishinaka T, Taenaka Y, Nakata M, Takano H. Prolonged nonpulsatile left heart bypass diminishes vascular contractility. Int J Artif Organs. 1999;22:492-8.

37. Roumy A, Liaudet L, Rusca M, Marcucci C, Kirsch M. Pulmonary complications associated with veno-arterial extra-corporeal membrane oxygenation: a comprehensive review. Crit Care. 2020;24: 212.

38. Langer T, Santini A, Bottino N, et al. "Awake" extracorporeal membrane oxygenation (ECMO): pathophysiology, technical considerations, and clinical pioneering. Crit Care. 2016;20:150.

39. Stevens MC, Callaghan FM, Forrest P, Bannon PG, Grieve SM. Flow mixing during peripheral veno-arterial extra corporeal membrane oxygenation - A simulation study. J Biomech. 2017;55:64 70.

40. Jett GK. Physiology of nonpulsatile circulation: acute versus chronic support. ASAIO J. 1999;45:119-22.

41. Saito S, Nishinaka T. Chronic nonpulsatile blood flow is compatible with normal end-organ function: implications for LVAD development. J Artif Organs. 2005;8:143-8.

42. Butt W, Heard M, Peek GJ. Clinical management of the extracorporeal membrane oxygenation circuit. Pediatr Crit Care Med. 2013;14:S13-9.

43. Hastings SM, Ku DN, Wagoner S, Maher KO, Deshpande S. Sources of circuit thrombosis in pediatric extracorporeal membrane oxygenation. ASAIO J. 2017;63:86-92.

44. Lo Coco V, Lorusso R, Raffa GM, et al. Clinical complications during veno-arterial extracorporeal membrane oxigenation in post-cardiotomy and non post-cardiotomy shock: still the Achilles' heel. J Thorac Dis. 2018;10:6993-7004.

45. Thiagarajan RR, Barbaro RP, Rycus PT, et al. Extracorporeal life support organization registry international report 2016. ASAIO J. 2017;63:60-7.

46. Cheng R, Hachamovitch R, Kittleson M, et al. Complications of extracorporeal membrane oxygenation for treatment of cardiogenic shock and cardiac arrest: a meta-analysis of 1,866 adult patients. Ann Thorac Surg. 2014;97:610-6. 
47. Martucci G, Panarello G, Occhipinti G, et al. Anticoagulation and transfusions management in veno-venous extracorporeal membrane oxygenation for acute respiratory distress syndrome: assessment of factors associated with transfusion requirements and mortality. J Intensive Care Med. 2019;34:630-9.

48. Cuker A. Recent advances in heparin-induced thrombocytopenia. Curr Opin Hematol. 2011;18:315-22.

49. Choi JH, Luc JGY, Weber MP, et al. Heparin-induced thrombocytopenia during extracorporeal life support: incidence, management and outcomes. Ann Cardiothorac Surg. 2019;8:19-31.

50. Pagano D, Milojevic M, Meesters MI, et al. 2017 EACTS/EACTA guidelines on patient blood management for adult cardiac surgery. Eur J Cardiothorac Surg. 2018;53:79-111.

51. Amata M, Martucci G, Granata A, et al. The role of endoscopy as non-invasive procedure to manage gastrointestinal complications during extracorporeal membrane oxygenation. Perfusion. 2020;267659120909669.

52. El Banayosy AM, Koerner MM, Harper M, et al. Gastrointestinal bleeding in ECMO patients: incidence and outcomes. J Heart Lung Transplant. 2020;39:S425.

53. Mazzeffi M, Greenwood J, Tanaka K, et al. Bleeding, transfusion, and mortality on extracorporeal life support: ECLS Working Group on Thrombosis and Hemostasis. Ann Thorac Surg. 2016;101:682-9.

54. Alhazzani W, Alshamsi F, Belley-Cote E, et al. Efficacy and safety of stress ulcer prophylaxis in critically ill patients: a network metaanalysis of randomized trials. Intensive Care Med. 2018;44:1-11.

55. Freundt M, Lunz D, Philipp A, et al. Impact of dynamic changes of elevated bilirubin on survival in patients on veno-arterial extracorporeal life support for acute circulatory failure. PLoS One. 2017;12: e0184995.

56. Lightsey JM, Rockey DC. Current concepts in ischemic hepatitis. Curr Opin Gastroenterol. 2017;33:158-63.

57. Roth C, Schrutka L, Binder C, et al. Liver function predicts survival in patients undergoing extracorporeal membrane oxygenation following cardiovascular surgery. Crit Care. 2016;20:57.

58. Kaestner F, Rapp D, Trudzinski FC, et al. High serum bilirubin levels, NT-pro-BNP, and lactate predict mortality in Long-term, severely ill respiratory ECMO patients. ASAIO J. 2018;64:232-7.

59. Cho YH, Yang J-H, Sung K, et al. Extracorporeal life support as a bridge to heart transplantation: importance of organ failure in recipient selection. ASAIO J. 2015;61:139-43.

60. Sern LH. Baseline MELD-XI score and outcome from veno-arterial extracorporeal membrane oxygenation support for acute decompensated heart failure. Eur Heart J Acute Cardiovasc Care. 2016;5:82-8.

61. Mazzeffi M, Kon Z, Sanchez P, Herr D. Impact of acute liver failure on mortality during adult ECLS. Intensive Care Med. 2016;42:299_ 300.

62. Poptsov V, Spirina E, Dogonasheva A, Zolotova E. Five years' experience with a peripheral veno-arterial ECMO for mechanical bridge to heart transplantation. J Thorac Dis. 2019;11:S889-901.

63. Sparks BE, Cavarocchi NC, Hirose H. Extracorporeal membrane oxygenation with multiple-organ failure: can molecular adsorbent recirculating system therapy improve survival? J Heart Lung Transplant. 2017;36:71-6.

64. Ostermann M, Connor M, Kashani K. Continuous renal replacement therapy during extracorporeal membrane oxygenation: why, when and how? Curr Opin Crit Care. 2018;24:493-503.

65. Han S-S, Kim HJ, Lee SJ, et al. Effects of renal replacement therapy in patients receiving extracorporeal membrane oxygenation: a meta-analysis. Ann Thorac Surg. 2015;100:1485-95.

66. Paden ML, Warshaw BL, Heard ML, Fortenberry JD. Recovery of renal function and survival after continuous renal replacement therapy during extracorporeal membrane oxygenation. Pediatr Crit Care Med. 2011;12:153-8.
67. Le Guennec L, Cholet C, Huang F, et al. Ischemic and hemorrhagic brain injury during venoarterial-extracorporeal membrane oxygenation. Ann Intensive Care. 2018;8:129.

68. Sutter R, Tisljar K, Marsch S. Acute neurologic complications during extracorporeal membrane oxygenation: a systematic review. Crit Care Med. 2018;46:1506-13.

69. Polito A, Barrett CS, Wypij D, et al. Neurologic complications in neonates supported with extracorporeal membrane oxygenation. An analysis of ELSO registry data. Intensive Care Med. 2013;39: 1594-601.

70. Organization ELS. ECLS registry report. Organization ELS; 2020. Available at: https://www.elso.org/Portals/0/Files/Reports/2020 January/International\%20Summary\%20January\%202020_page1. pdf.

71. Bizzarro MJ, Conrad SA, Kaufman DA, Rycus P. Infections acquired during extracorporeal membrane oxygenation in neonates, children, and adults. Pediatr Crit Care Med. 2011;12:277-81.

72. Cashen K, Reeder R, Dalton HJ, et al. Acquired infection during neonatal and pediatric extracorporeal membrane oxygenation. Perfusion. 2018;33:472-82.

73. Schmidt M, Bréchot N, Hariri S, et al. Nosocomial infections in adult cardiogenic shock patients supported by venoarterial extracorporeal membrane oxygenation. Clin Infect Dis. 2012;55:1633-41.

74. Castagnola E, Gargiullo L, Loy A, et al. Epidemiology of infectious complications during extracorporeal membrane oxygenation in children: a single-center experience in 46 runs. Pediatr Infect Dis J. 2018;37:624-6.

75. Aziz F, Brehm CE, El-Banyosy A, Han DC, Atnip RG, Reed AB. Arterial complications in patients undergoing extracorporeal membrane oxygenation via femoral cannulation. Ann Vasc Surg. 2014;28:178-83.

76. Lamarche Y, Chow B, Bédard A, et al. Thromboembolic events in patients on extracorporeal membrane oxygenation without anticoagulation. Innovations (Phila). 2010;5:424-9.

77. Alexis-Ruiz A, Ghadimi K, Raiten J, et al. Hypoxia and complications of oxygenation in extracorporeal membrane oxygenation. J Cardiothorac Vasc Anesth. 2019;33:1375-81.

78. Mascio CE, Austin EH, Jacobs JP, et al. Perioperative mechanical circulatory support in children: an analysis of the Society of Thoracic Surgeons Congenital Heart Surgery Database. J Thorac Cardiovasc Surg. 2014;147:658-64.

79. Bartlett RH. Physiology of gas exchange during ECMO for respiratory failure. J Intensive Care Med. 2017;32:243-8.

80. Risch L, Fischer JE, Herklotz R, Huber AR. Heparin-induced thrombocytopenia in paediatrics: clinical characteristics, therapy and outcomes. Intensive Care Med. 2004;30:1615-24.

81. Pediatric cardiac failure, Extracorporeal Life Support Organization, Ann Arbor, MI. [cited 2017 Feb 15]. Available from http://www. elso.org/resources/guidelines.aspx.

82. Callahan R, Trucco SM, Wearden PD, Beerman LB, Arora G, Kreutzer J. Outcomes of pediatric patients undergoing cardiac catheterization while on extracorporeal membrane oxygenation. Pediatr Cardiol. 2015;36:625-32.

83. Bautista-Rodriguez C, Sanchez-de-Toledo J, Da Cruz EM. The role of echocardiography in neonates and pediatric patients on extracorporeal membrane oxygenation. Front Pediatr. 2018;6:297.

84. Marino BS, Tabbutt S, MacLaren G, et al. Cardiopulmonary resuscitation in infants and children with cardiac disease: a scientific statement from the American Heart Association. Circulation. 2018;137:e691-782.

85. Kilburn DJ, Shekar K, Fraser JF. The complex relationship of extracorporeal membrane oxygenation and acute kidney injury: causation or association? Biomed Res Int. 2016;2016:1094296.

86. Kolovos NS, Bratton SL, Moler FW, et al. Outcome of pediatric patients treated with extracorporeal life support after cardiac surgery. Ann Thorac Surg. 2003;76:1435-41. 
87. Ong C, Mok YH, Tan ZH, et al. Nutritional practices and adequacy in children supported on extracorporeal membrane oxygenation. Clin Nutr ESPEN. 2018;26:21-6.

88. Mehta NM, Skillman HE, Irving SY, et al. Guidelines for the provision and assessment of nutrition support therapy in the pediatric critically ill patient: Society of Critical Care Medicine and American Society for Parenteral and Enteral Nutrition. Pediatr Crit Care Med. 2017;18:675-715.

89. Pandya NR, Daley M, Mattke A, et al. A comparison of pumpcontrolled retrograde trial off to arterio-venous bridging for weaning from venoarterial extracorporeal membrane oxygenation. Eur J Cardiothorac Surg. 2019. https://doi.org/10.1093/ejcts/ ezy485.

90. Jaggers JJ, Forbess JM, Shah AS, et al. Extracorporeal membrane oxygenation for infant postcardiotomy support: significance of shunt management. Ann Thorac Surg. 2000;69:1476-83.

91. Jolley M, Colan SD, Rhodes J, DiNardo J. Fontan physiology revisited. Anesth Analg. 2015;121:172-82.

92. Guihaire J, Dang Van S, Rouze S, et al. Clinical outcomes in patients after extracorporeal membrane oxygenation support for postcardiotomy cardiogenic shock: a single-centre experience of 92 cases. Interact Cardiovasc Thorac Surg. 2017;25:363-9.

93. Lai Y, Ortoleva J, Villavicencio M, et al. Outcomes of venoarterial extracorporeal membrane oxygenation patients requiring multiple episodes of support. J Cardiothorac Vasc Anesth. 2020;34:235761.
94. von Bahr V, Hultman J, Eksborg S, Frenckner B, Kalzén H. Longterm survival in adults treated with extracorporeal membrane oxygenation for respiratory failure and sepsis. Crit Care Med. 2017;45: 164-70.

95. de Waha S, Fuernau G, Eitel I, Desch S, Thiele H. Long-term prognosis after extracorporeal life support in refractory cardiogenic shock - results from a real-world cohort. EuroIntervention. 2016;12:414.

96. Holland R, Rechel B, Stepien K, Harvey I, Brooksby I. Patients' self-assessed functional status in heart failure by New York Heart Association class: a prognostic predictor of hospitalizations, quality of life and death. J Card Fail. 2010;16:150-6.

97. Chen S-W, Tsai F-C, Lin Y-S, et al. Long-term outcomes of extracorporeal membrane oxygenation support for postcardiotomy shock. J Thorac Cardiovasc Surg. 2017;154:469-477.e2.

98. Chiu R, Pillado E, Sareh S, De La Cruz K, Shemin RJ, Benharash P. Financial and clinical outcomes of extracorporeal mechanical support. J Card Surg. 2017;32:215-21.

99. Birks EJ. The comparative use of ventricular assist devices: differences between Europe and the United States. Tex Heart Inst J. 2010;37:565-7.

Publisher's note Springer Nature remains neutral with regard to jurisdictional claims in published maps and institutional affiliations. 